\title{
Avaliação de características produtivas em animais da raça Nelore por meio de análise multivariada
}

\section{Use of multivariate analysis to evaluate productive traits in Nellore cattle}

\author{
Júlio César de Souza, ${ }^{*}$ Fernando Marques Salles, ${ }^{* *}$ Luiz Otávio Campos da Silva, ${ }^{* * *}$ Marcelo Falci Mota, ${ }^{* *}$

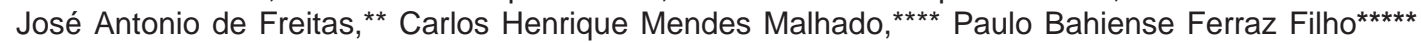

\begin{abstract}
Resumo
O objetivo deste estudo foi avaliar as principais características zootécnicas produtivas e suas correlações fenotípicas entre estas como peso ao nascer (PN), peso aos 120 (P120), aos 180 (P180), aos 205 (P205), aos 240 (P240), aos 300 (P300), aos 365 (P365), aos 420 (P420), aos 480 (P480), aos 540 (P540) dias de idade; ganhos de peso do nascimento até os 120 (GPN120), aos 205 (GPN205) dias de idade e o peso dos 120 aos 205 (GP120_205), dos 205 aos 365 (GP205_365) e dos 365 aos 540 (GP365_540) dias de idade e estimar a influência da idade da vaca em 12.440 bezerros da raça Nelore nascidos no estado de Minas Gerais, usando técnicas de análises multivariadas. O menor $(0,101)$ valor encontrado para as correlações foi entre P205_365 e P365_540 e o maior $(0,996)$ foi entre P205 e PN205. Os cinco primeiros componentes principais justificaram $97,08 \%$, sendo que o primeiro justificou $63,51 \%$, o segundo $12,1 \%$ e o terceiro $8,3 \%$ e os outros dois, $13,17 \%$, respectivamente. O maior peso observado aos 240 dias, $194,1 \mathrm{~kg}$, ocorreu em bezerros nascidos de vacas com 108 meses de idade; o menor peso observado foi de 169,5kg para bezerros nascidos de vacas com 240 meses de idade.
\end{abstract}

Palavras-chave: principal componente, peso, zebu.

\begin{abstract}
The objective of this study was to evaluate the principal components and correlations between the weight at birth (BWT), at 120 (WT120), at 180 (WT180), at 205 (WT205), at 240 (WT240), at 300 (WT300), at 365 (WT365), at 420 (WT420), at 480 (WT480) and at 540 (WT540) days of age; gains of weight from birth to 120 (BWT120), to 205 (BWT205) days of age and the weight from 120 to 205 (WT120_205), from 205 to 365 (WT205_365) and from 365 to 540 (WT365_540) days of age and influence of the age of dam of 12.440 animals of Nellore breed born in the State of Minas Gerais, using multivariate analysis tecnics. The lower value of correlation was between WT205_365 and WT365 540, 0,101; the higher value of correlation was between WT205 and BWT205, 0,996. The first five principal components justify $97,08 \%$, since the first justify $63,51 \%$, the second $12,1 \%$ and the third $8,3 \%$ and the others two, $13.17 \%$. The higher value at 240 days, $194,1 \mathrm{~kg}$, was for calves born from 108 months dams; the lower weight was $169,5 \mathrm{~kg}$ for calves born from 240 months dams.
\end{abstract}

Keywords: principal component, weight, zebu.

\section{Introdução}

Um importante grupo de características zootécnicas em bovinos de corte é aquele ligado à produção, dentre estes o peso em diferentes idades, sendo muito utilizado por ser de fácil obtenção, garantindo uma avaliação exata do crescimento do animal e apresentando herdabilidade média a alta, sugerindo elevado progresso genético ao selecionar para essas características. Algumas delas são correlacionadas entre si, indicando que, em geral, respondem bem à seleção e que a seleção para qualquer um deles deve provocar mudanças nos outros, na mesma direção (Alencar, 2002).
A correlação entre características pode ser analisada através dos componentes principais, uma técnica estatística de análise multivariada, que transforma linearmente um grupo de variáveis num conjunto substancialmente menor, de variáveis correlacionadas, que representam a maior parte da informação do conjunto original (Silva e Padovani, 2006). O principal componente representa o maior eixo de variação entre as observações no espaço multidimensional; o segundo principal componente mostra a menor variação entre as observações (Becker, 1988, Timm, 2002). Análises utilizando componentes principais é uma combinação linear de um conjunto de co-variáveis que permitem identificar as

\footnotetext{
* Professor da Universidade Federal do Mato Grosso do Sul, Campus Aquidauana, DBA, UFMS, MS. E-mail: jcs@cpaq.ufms.br.

** Professor da Universidade Federal do Paraná, Campus Palotina - UFPR.

*** Pesquisador da Embrapa, CNPGC, Campo Grande, MS.

${ }^{* * * *}$ Professor DCB, Campus Três Lagoas, UFMS, MS.

${ }^{* * * * *}$ Professor da DCB, Campus Jequié, UESB, BA.
} 
variáveis que mais contribuem na avaliação. Souza et al. (2010) reportaram que o uso de análise multivariada para avaliação de dados pode ser uma ferramenta a mais para auxiliar no melhoramento genético. Estimativas de correlações, distâncias genéticas e de índices utilizando resultados a partir desse tipo de análise pode promover bons resultados em programas de melhoramento. Através da análise de componentes principais pode-se obter a matriz de variância e covariância ou a matriz de correlação.

Ferraz Filho et al. (2006) reportaram que o primeiro componente principal explicou uma mudança de $30,33 \%$, o segundo $24,09 \%$ e o terceiro $16,75 \%$. Souza et al. (2007a) avaliando o desenvolvimento de búfalos criados em diferentes regiões brasileiras utilizando análise multivariada verificaram valores de eigenvalues dos primeiros quatro componentes principais iguais a 5,29, 2,54, 1,66, 1,01, respectivamente; estes justificaram 95\%. Mascioli et al. (2000), avaliando parâmetros genéticos de bovinos de corte, constataram que o primeiro componente principal justificou $74 \%$ da variação total. Souza et al. (2007b), avaliando animais Hereford, utilizando análise multivariada encontraram valores iguais a 5,33, 3,75 e 2,03, para os eigenvalues e os três componentes principais juntos justificaram $86 \%$.

Gemin et al. (2004), avaliando o efeito do meio e da idade da vaca sobre o ganho de peso na fase de cria, recria e os dias para se obter $160 \mathrm{~kg}$ em animais da raça Guzerá, verificaram que o efeito da idade da vaca mostrou-se significativo $(P<$ $0,05)$ nas características que envolvem a fase de cria. Essa teve sua fase ótima, quando suas progênies desmamaram mais pesadas aos 95 meses de idade e 93 meses, respectivamente para D160 e GPD, demonstrando que a partir desse ponto o peso das progênies começam a cair, indicando que a matriz já pode ser descartada.

A influência da idade da vaca deve ser considerada num programa de melhoramento genético, pois interfere no ganho de peso dos bezerros e, segundo Gemin et al. (2004) e Souza et al. (2004), são necessários ajustes aditivos para a idade da vaca ao parto.

O objetivo deste trabalho foi estimar componentes principais, correlações entre o PN, peso aos 205, 365, 550 e 730 dias de idade, ganhos de peso do nascer aos 120 (PN120), aos 205 (PN205) dias de idade e o peso dos 120 aos 205 (P105_205), dos 205 aos 365 (P205_365) e dos 365 aos 540 (P365_540) dias de idade, o ganho médio diário do animal do nascimento à desmama e as distâncias entre os grupos genéticos de vacas.

\section{Material e métodos}

Os dados usados são provenientes do arquivo do Controle de Desenvolvimento Ponderal da Associação Brasileira de Criadores de Zebu (ABCZ). As informações referem-se a pesagens padronizadas de animais da raça Nelore, nascidos no estado de Minas Gerais, no período de 1975 a 2001, totalizando 9.408 matrizes, locadas em 174 fazendas. As características avaliadas foram o peso ao nascer (PN), aos 120 (P120), aos 180 (P180), aos 205 (P205), aos 240 (P240), aos 300 (P300), aos 365 (P365), aos 420 (P420), aos 480 (P480) e aos 540 (P540) dias de idade; ganhos de peso do nascer aos 120 (PN120), aos 205 (PN205) dias de idade e o peso dos 120 aos 205 (P105_205), dos 205 aos
365 (P205 365) e dos 365 aos 540 (P365 540) dias de idade. Calculou-se também o ganho médio diário do animal do nascimento à desmama (GMDd = [peso à desmama peso ao nascer] / idade do animal a desmama (em dias)) .

Os pesos aos 120 e 205 dias de idade foram ajustados multiplicando-se o GMDd pela idade e somando-se o peso ao nascer, $\mathrm{P} 120=$ [ GMDd ajustado $\times 120$ dias $]+$ peso ao nascer); P205 = [ GMDd ajustado x 205 dias ] + peso ao nascer). Para P365 e P540 calcula-se o GMDpa - ganho médio diário pós-desmama acumulado, que é a média do GMDd e do GMDp - ganho médio diário pós-desmama (GMDp $=$ [pesagem atual - P205] / idade do animal em dias na pesagem pós-desmama - 205). Uma vez obtido o GMDpa, calculam-se os P365 e P540 por meio das seguintes fórmulas: P365 = P205 ajustado + (GMDpa x 160 dias) e P540 = P205 ajustado + (GMDpa x 345 dias).

Os componentes principais foram estimados usando o procedimento PRINCOMP; os efeitos da idade vaca sobre os pesos aos 205 e 240 dias foram obtidos por regressão quadrática da idade da vaca sobre o peso do bezerro.

\section{Resultados e discussão}

Os valores da média e desvio-padrão obtidos pelo procedimento Princomp (SAS, 2007) encontram-se na Tabela 1. Utilizando-se 18 variáveis na análise, apenas 2.289 animais atenderam a todas as variáveis. Uma vez rodadas as análises, eliminaram-se aquelas variáveis com $n$ menor e repetiu-se o procedimento, porém, com 15 variáveis e um aumento para 12.440 animais. Isso demonstra a necessidade de se escolher somente os dados mais relevantes para que se trabalhe apenas com as características que realmente contribuam no objetivo do estudo.

Em se tratando de dados de campo, isso possibilita um universo maior de informações, bem como selecionar as características mais importantes, permitindo a redução do número de informações coletadas, facilitando, assim, a confiabilidade e precisão dos dados. A matriz de correlação entre as características produtivas de animais da raça Nelore mostra diferentes graus de correlação (Tabela 2).

O maior valor de correlação observado foi 0,996 , entre as características P205 e GPN205, seguido por 0,992 para P120 e PN120, enquanto o menor valor de correlação observado foi 0,101 , obtido para a correlação entre GP205_365e GP365_540. Quando se compararam as correlações obtidas entre o GPN_205 com o peso aos 365, a correlação foi de 0,761 e o $P 5 \overline{4} 0$ foi igual a 0,699 .

O conjunto de autovetores permitiu a visualização das variáveis que mais influenciaram os componentes principais. As variáveis que mais influenciaram foram a P240 e PN, respectivamente, o maior e menor valor. Com base no primeiro componente principal, poderíamos selecionar para avaliação P240 ou P205, P365, visto que o P300 estaria muito próximo do P240, ainda indicaria o P420 ou 540, ambas tiveram valores próximos (Tabela 3 ), além de inserir na avaliação o GPN205. Os resultados obtidos aqui estão próximos aos reportados por Ferraz Filho et al. (2006), Souza et al. (2007 a,b) e Souza et al. (2010).

Os dois primeiros componentes principais já justificaram $75,61 \%$. Quando se tomaram os cinco primeiros componen- 
Tabela 1: Valores de média e desvio-padrão, obtidos pelo procedimento Princomp de animais Nelore, criados em Minas Gerais

\begin{tabular}{cccccccc}
\hline PN & P120 & P180 & P205 & P240 & P300 & P365 & P420 \\
\hline $30,1 \pm 2,8$ & $119,1 \pm 21,6$ & $157,8 \pm 28,3$ & $174,1 \pm 32,6$ & $190,4 \pm 36,4$ & $212,6 \pm 42,8$ & $235,2 \pm 48,2$ & $256,4 \pm 55,4$ \\
\hline P480 & P540 & PN120 & GPN205 & GP120_205 & P480 & GP365_540 \\
\hline $282,1 \pm 62,49$ & $309,27 \pm 68,79$ & $0,741 \pm 0,174$ & $0,702 \pm 0,155$ & $0,647 \pm 0,250$ & $0,381 \pm 0,194$ & $0,423 \pm 0,214$ &
\end{tabular}

Peso ao nascer (PN), aos 120 (P120), aos 180 (P180), aos 205 (P205), aos 240 (P240), aos 300 (P300), aos 365 (P365), aos 420 (P420), aos 480 (P480) e aos 540 (P540) dias de idade; ganhos de peso do nascer aos 120 (PN120), aos 205 (PN205) dias de idade e o peso dos 120 aos 205 (P105_205), dos 205 aos 365 (P205_365) e dos 365 aos 540 (P365_540) dias de idade.

Tabela 2: Matriz de correlação entre as características produtivas de animais da raça Nelore criados em Minas Gerais

\begin{tabular}{|c|c|c|c|c|c|c|c|c|c|c|c|c|c|c|}
\hline & $\frac{\text { D }}{\tilde{O}}$ & $\underset{\text { o }}{\stackrel{0}{0}}$ & 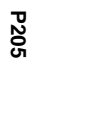 & $\begin{array}{l}\text { D̃ } \\
\text { I }\end{array}$ & ठ্ట్ & 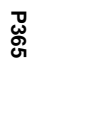 & 完 & $\begin{array}{l}\stackrel{0}{0} \\
\stackrel{0}{\circ}\end{array}$ & $\begin{array}{l}\text { D্ } \\
\text { ثै }\end{array}$ & $\frac{\text { Z }}{\frac{N}{O}}$ & 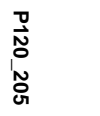 & $\begin{array}{l}\text { Do } \\
\text { 'N } \\
\text { No }\end{array}$ & 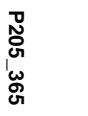 & 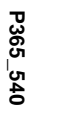 \\
\hline PN & 0,279 & 0,287 & 0,276 & 0,272 & 0,266 & 0,286 & 0,283 & 0,284 & 0,297 & 0,153 & 0,140 & 0,193 & 0,154 & 0,177 \\
\hline P120 & 1,000 & 0,859 & 0,765 & 0,696 & 0,599 & 0,598 & 0,588 & 0,588 & 0,568 & 0,992 & 0,160 & 0,757 & 0,124 & 0,273 \\
\hline P180 & & 1,000 & 0,947 & 0,883 & 0,770 & 0,745 & 0,702 & 0,694 & 0,696 & 0,845 & 0,581 & 0,941 & 0,162 & 0,319 \\
\hline P205 & & & 1,000 & 0,941 & 0,811 & 0,770 & 0,714 & 0,700 & 0,710 & 0,751 & 0,758 & 0,996 & 0,145 & 0,313 \\
\hline P240 & & & & 1,000 & 0,905 & 0,830 & 0,754 & 0,730 & 0,743 & 0,680 & 0,737 & 0,936 & 0,300 & 0,296 \\
\hline P300 & & & & & 1,000 & 0,923 & 0,814 & 0,769 & 0,775 & 0,581 & 0,636 & 0,805 & 0,581 & 0,234 \\
\hline P365 & & & & & & 1,000 & 0,938 & 0,868 & 0,852 & 0,577 & 0,575 & 0,761 & 0,743 & 0,277 \\
\hline P420 & & & & & & & 1,000 & 0,922 & 0,861 & 0,567 & 0,499 & 0,704 & 0,705 & 0,374 \\
\hline P480 & & & & & & & & 1,000 & 0,888 & 0,567 & 0,478 & 0,690 & 0,612 & 0,513 \\
\hline P540 & & & & & & & & & 1,000 & 0,545 & 0,514 & 0,699 & 0,576 & 0,739 \\
\hline PN120 & & & & & & & & & & 1,000 & 0,146 & 0,753 & 0,107 & 0,258 \\
\hline GP120_205 & & & & & & & & & & & 1,000 & 0,761 & 0,966 & 0,203 \\
\hline GPN_205 & & & & & & & & & & & & 1,000 & 0,135 & 0,304 \\
\hline GP205_365 & & & & & & & & & & & & & 1,000 & 0,101 \\
\hline
\end{tabular}

Peso ao nascer (PN), aos 120 (P120), aos 180 (P180), aos 205 (P205), aos 240 (P240), aos 300 (P300), aos 365 (P365), aos 420 (P420), aos 480 (P480) e aos 540 (P540) dias de idade; ganhos de peso do nascer aos 120 (PN120), aos 205 (PN205) dias de idade e o peso dos 120 aos 205 (P105_205), dos 205 aos 365 (P205_365) e dos 365 aos 540 (P365_540) dias de idade.

Tabela 3: Estimativas de autovalores $\left(\lambda_{\mathrm{J}}\right)$ obtidos a partir da matriz de correlação em pesos de animais da raça Nelore criados em Minas Gerais

\begin{tabular}{cccc}
\hline $\begin{array}{c}\text { Principal } \\
\text { componente (prin) }\end{array}$ & $\begin{array}{c}\text { Contribuição } \\
\left(\lambda_{J}\right)\end{array}$ & $\begin{array}{c}\text { Porcentagem } \\
\left(\lambda_{J}\right)\end{array}$ & $\begin{array}{c}\text { Porcentagem } \\
\text { acumulada }\left(\lambda_{J}\right)\end{array}$ \\
\hline Prin 1 & 9,52 & 63,51 & 63,51 \\
Prin 2 & 1,81 & 12,10 & 75,61 \\
Prin 3 & 1,24 & 8,30 & 83,91 \\
Prin 4 & 1,07 & 7,20 & 91,11 \\
Prin 5 & 0,89 & 5,97 & 97,08 \\
Prin 6 & 0,21 & 1,41 & 98,49 \\
Prin 7 & 0,09 & 0,65 & 99,14 \\
Prin 8 & 0,05 & 0,38 & 99,52 \\
Prin 9 & 0,04 & 0,28 & 99,80 \\
Prin 10 & 0,03 & 0,20 & 100,0 \\
\hline
\end{tabular}

tes principais, prin 1 a prin 5 , explicaram $97,08 \%$ da variação encontrada nos dados originais, sendo possível avaliar, por meio dos autovalores, a importância relativa de cada característica, bem como a contribuição em porcentagem de cada uma (Tabela 4).

As vacas com idade entre 60 e 156 meses de idade produziram bezerros com peso acima da média. Os bezerros com média de peso mais elevados aos 240 dias (P240) pesaram 194,1 $\mathrm{kg}$ (animais filhos de vacas com idade igual a 108 meses). Essas também geraram bezerros com maior ganho diário do nascer aos 205 dias (GN205), com média de $0,716 \mathrm{~kg} / \mathrm{dia}$. Os menores pesos de bezerros, observados aos 240 dias de idade (P240), foram os filhos de vacas com idade de 240 meses.

Essas também apresentaram os bezerros com menor ganho diário do nascer aos 205 dias (GN205), com média de 0,605 $\mathrm{kg} /$ dia (Tabela 5). A idade das matrizes nesse trabalho encontra-se 12 meses mais velha quando comparado com os resultados de Gemin et al. (2004).

As Figuras 1 e 2 mostram a distribuição para os pesos de bezerros da raça Nelore aos 205 e 240 dias de idade em Minas Gerais. 
Tabela 4: Conjunto de autovetores obtidos na matriz de correlação entre as variáveis avaliadas em animais da raça Nelore, criados em Minas Gerais

\begin{tabular}{lcccccc}
\hline \multicolumn{7}{c}{ Autovetores } \\
\hline P240 & Prin1 & Prin2 & Prin3 & Prin4 & Prin5 & Prin6 \\
P205 & 0,303181 & $-0,112812$ & $-0,186352$ & $-0,016799$ & 0,031957 & 0,288606 \\
P365 & 0,301071 & $-0,234745$ & $-0,150365$ & 0,045054 & 0,032477 & $-0,075259$ \\
GPN205 & 0,299445 & 0,226600 & $-0,067468$ & $-0,186564$ & 0,001951 & 0,052551 \\
P180 & 0,297984 & $-0,245741$ & $-0,169641$ & 0,032123 & $-0,050599$ & $-0,075991$ \\
P300 & 0,295935 & $-0,254364$ & 0,031268 & $-0,027631$ & 0,022582 & $-0,059775$ \\
P420 & 0,294343 & 0,101780 & $-0,165566$ & $-0,166540$ & 0,028159 & 0,550348 \\
P540 & 0,289210 & 0,255334 & 0,038162 & $-0,096432$ & $-0,040145$ & $-0,445327$ \\
P480 & 0,286218 & 0,229399 & 0,127746 & 0,276707 & $-0,114492$ & 0,151649 \\
P120 & 0,284602 & 0,232583 & 0,104788 & 0,060305 & $-0,079470$ & $-0,533464$ \\
GPN120 & 0,250666 & $-0,300347$ & 0,399122 & $-0,185812$ & 0,004245 & 0,021695 \\
GP120_205 & 0,243757 & $-0,318437$ & 0,386031 & $-0,212333$ & $-0,123324$ & 0,023575 \\
GP205_365 & 0,207592 & $-0,055457$ & $-0,635490$ & 0,257582 & 0,045513 & $-0,137453$ \\
GP365_540 & 0,148536 & 0,597591 & 0,053099 & $-0,336546$ & $-0,031039$ & 0,160425 \\
PN & 0,139930 & 0,129403 & 0,321365 & 0,748138 & $-0,212689$ & 0,210736 \\
\hline Pes0 00 & 0,105877 & 0,070073 & 0,184193 & 0,157843 & 0,953689 & $-0,009344$ \\
\hline
\end{tabular}

Peso ao nascer (PN), aos 120 (P120), aos 180 (P180), aos 205 (P205), aos 240 (P240), aos 300 (P300), aos 365 (P365), aos 420 (P420), aos 480 (P480) e aos 540 (P540) dias de idade; ganhos de peso do nascer aos 120 (PN120), aos 205 (PN205) dias de idade e o peso dos 120 aos 205 (P105_205), dos 205 aos 365 (P205_365) e dos 365 aos 540 (P365_540) dias de idade.

Tabela 5: Relação entre o peso aos 240 dias (P240) e o ganho diário do peso ao nascer até os 205 dias de idade (GN205) em bezerros Nelore nascidos em diferentes idades da vaca em Minas Gerais

\begin{tabular}{ccc}
\hline $\begin{array}{c}\text { Idade da vaca } \\
\text { (meses) }\end{array}$ & $\begin{array}{c}\text { P240 } \\
\text { (kg) }\end{array}$ & $\begin{array}{c}\text { GN 205 } \\
\text { (kg/dia) }\end{array}$ \\
\hline 24 & 185,3 & 0,676 \\
36 & 187,7 & 0,687 \\
48 & 189,7 & 0,696 \\
60 & 191,4 & 0,704 \\
72 & 192,7 & 0,709 \\
84 & 193,6 & 0,713 \\
96 & 194,0 & 0,715 \\
108 & 194,1 & 0,716 \\
120 & 193,9 & 0,714 \\
132 & 193,2 & 0,711 \\
144 & 192,1 & 0,706 \\
156 & 190,6 & 0,700 \\
168 & 188,8 & 0,692 \\
180 & 186,5 & 0,681 \\
192 & 183,9 & 0,670 \\
204 & 180,9 & 0,656 \\
216 & 177,5 & 0,641 \\
228 & 173,7 & 0,624 \\
240 & 169,5 & 0,605 \\
\hline
\end{tabular}

Peso ao nascer (PN), aos 120 (P120), aos 180 (P180), aos 205 (P205), aos 240 (P240), aos 300 (P300), aos 365 (P365), aos 420 (P420), aos 480 (P480) e aos 540 (P540) dias de idade; ganhos de peso do nascer aos 120 (PN120), aos 205 (PN205) dias de idade e o peso dos 120 aos 205 (P105_205), dos 205 aos 365 (P205_365) e dos 365 aos 540 (P365 540) dias de idade.

O peso aos 240 dias apresentou correlação elevada com P365, P420, P540 e GPN_205 mostrando ser útil para se fazer uma primeira seleção a essa idade. A idade da vaca influenciou o peso aos 240 dias (P240) e no ganho médio diário do nascer aos 205 dias (GN205), comprovando a necessidade de ajustes aditivos para a idade da vaca ao parto.

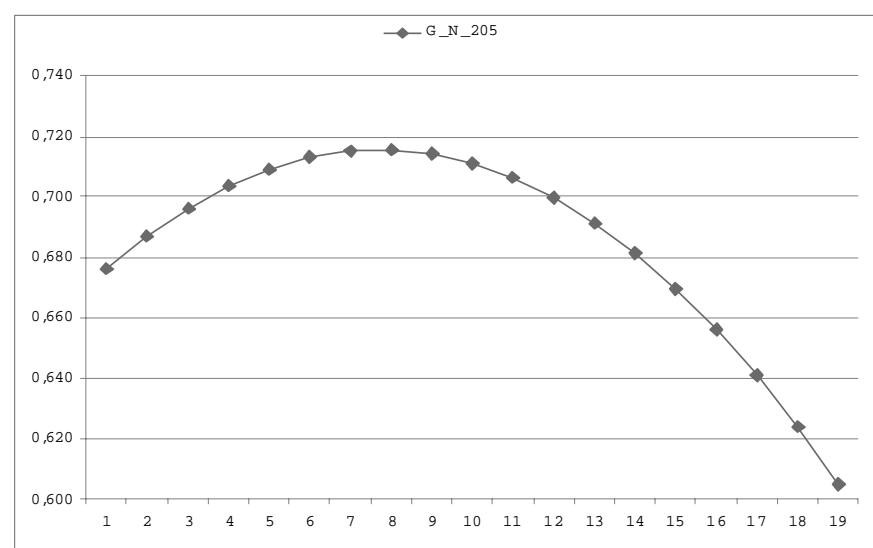

Figura 1: Relação entre a idade da vaca e o ganho em peso do nascimento aos 205 dias de idade de seus bezerros

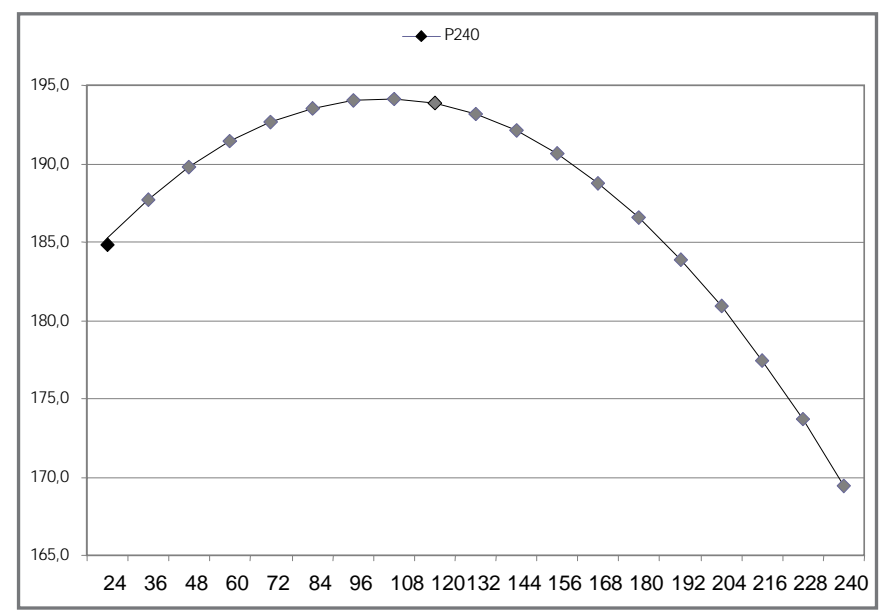

Figura 2: Relação entre a idade da vaca e o peso aos 240 dias de idade de seus bezerros 


\section{Conclusão}

O peso aos 240 dias apresentou correlação elevada com P365, P420, P540 e GPN_205 mostrando ser útil para se fazer uma primeira seleção a essa idade. A idade da vaca

\section{Referências}

ALENCAR, M.M. Critérios de seleção e a moderna pecuária bovina de corte brasileira. Anais do IV Simpósio Nacional de Melhoramento Animal, 2002.

BECKER, J.F., STEWART, T.S., LONG, CR, CARTWRIGHT, T.C. Multiple regression and principal components analysis of puberty and growth in cattle. Jour. of Anim. Sci. v. 9, p. 2147-2158. 1988.

FERRAZFILHO, P.B., SILVA, L.O.C., SOUZA, J. C., MALHADO, C.H.M. Divergência Genética de Touros Nelores com Sêmen Disponível em Centrais de Inseminação no Brasil. In: 43 Reunião Anual da Sociedade Brasileira de Zootecnia, 2006, João Pessoa. Anais... SBZ. Viçosa : SBZ, 2006. v. 1.

GEMIN, E., SOUZA, J.C., SILVA, L.O.C., MALHADO, C.H.M., FERRAZ FILHO, P.B. Efeito do meio e da idade da vaca sobre o ganho de peso na fase de cria, recria e os dias para se obter $160 \mathrm{~kg}$ em animais da raça Guzerá, Archives of Veterinary Science, v. 9, n. 1, p. 93-99, 2004.

MASCIOLI, A.S., EL FARO, L., ALENCAR, M.M., FRIES, L.A., BARBOSA, P.F. Genetic and Phenotypic Parameters and Principal Components Analyses for Body Weight Gains in Canchim Cattle, Brazilian Journal of Animal Science, v. 29, n. 6, p.1654-1660, 2000.

SILVA, N.R.; PADOVANI, C.R. Utilização de componentes principais em experimentação agronômica. Energia na Agricultura, Botucatu, v. 21 , n. 4 , p. $98-113,2006$. influenciou o peso aos 240 dias (P240) e no ganho médio diário do nascer aos 205 dias (GN205), comprovando a necessidade de ajustes aditivos para a idade da vaca ao parto.

SOUZA J.C., SILVA, L.O.C., MALHADO C.H.M., SERENO, J.R.B., FREITAS, J.A., SANTOS, I.W., FERRAZ FILHO, P.B., GONDO, A. Efeitos ambientais e genéticos sobre o peso ao desmame de animais da raça Nelore criados no Pantanal Sul-Mato-Grossense. In: Simpan 2004. IV Simpósio sobre recursos naturais e socioeconômicos do Pantanal. Corumbá, 2004. CD.

SOUZA, J.C., RAMOS, A. A., MALHADO, C.H. M. , JORGE, A.M. , FERRAZFILHO, P.B., DEFREITAS, J.A., FLOURNOY, N. , LAMBERSON, W.R. Evaluation of development traits of beef buffalo in Brazil using multivariate analysis, Italian Journal of Animal Science, v. 6, 2007. Supp. 2 - Part 2. p.321-324, 2007ㄹ.

SOUZA, J.C., CAMPOS, L.T., FREITAS, J.A., WEABER, R., LAMBERSON, W.R. Evaluation of Growth Traits of Brazilian Herefords using Multivariate Analysis. In: Annual Meeting of the American Society of Animal Science, 2007, San Antonio, TX. Anais... American Society of Animal Science. Champagnat: JAS, [2007b]. (CD-Rom).

SOUZA, J.C., PEROTTO, D., ABRAHÃO, J.J., FREITAS, J.A., FERRAZ FILHO, P.B., WEABER, R.L. E LAMBERSON, W.R. Estimativa das distâncias genéticas e componentes principais com animais cruzados no Brasil, usando análise multivariada. Arch. Zootec., v. 59, n. 228 p. 479-485, 2010.

TIMM, N.H. Applied Multivariate Analysis. New York. Springer-Verlag, 2002. 